\title{
Observatorio
}

\section{Información en la empresa para innovar y competir}

\section{Por Antonia Ferrer}

\begin{abstract}
Resumen: Aunque la innovación es un factor clave para la supervivencia de las empresas, su situación en España se encuentra aún lejos de los objetivos marcados por la Estrategia de Lisboa, por lo que las administraciones públicas ha lanzado varias medidas para potenciarla. Para poder innovar es esencial captar y seleccionar la mejor información posible, lo cual implica principalmente usar bases de datos científicas y técnicas, pero éstas son poco conocidas en el ámbito empresarial. El artículo hace un breve repaso al uso de información para la innovación en las empresas e inserta en el contexto mencionado las principales conclusiones del Informe Cotec 2008.
\end{abstract}

Palabras clave: Información en la empresa, Uso de la

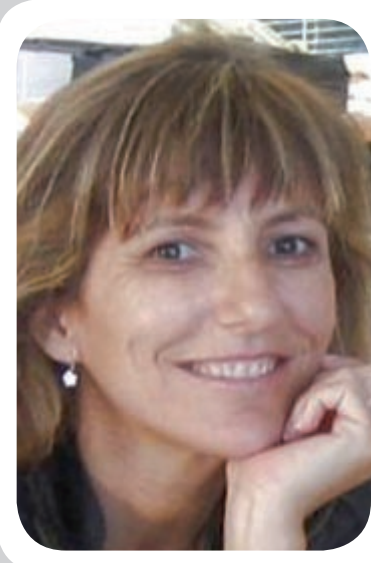
Antonia Ferrer-Sapena, licenciada en geografía e historia en la especialidad de contemporánea por la Universitat de Valencia y doctora en técnicas y métodos actuales en información y documentación, es coordinadora de investigación en Florida Centre de Formació y miembro del Comité de Innovación. Entre los últimos proyectos dirigidos destaca el portal de economía social EcSocial.com <http://www. ecsocial.com>. Es profesora de la Universidad Politécnica de Valencia, y miembro del foro Innovem Junts 2008 del Instituto de la Pequeña y Mediana Industria (Impiva) de la Generalitat Valenciana.

información, Innovación, I+D+I, Pequeña y mediana empresa, Pyme, Organismos financiadores, Política científica, España.

\section{Title: Information in companies as a tool to innovate and compete}

Abstract: Although innovation is a key factor for the survival of enterprises, its situation in Spain is still far from the objectives set by the Lisbon Strategy. As a consequence the Spanish public administrations have launched various measures to improve it. Innovation requires acquiring and selecting the best information available, which means mainly using scientific and technical databases, but they are little known in the business environment. The article gives a brief overview of the use of information for innovation in enterprises and comments on the main findings of 2008 Cotec Report.

Keywords: Information in companies, Information use, Innovation, $R \& D$, Small and medium size enterprises, Business information, SME, Funding agencies, Scientific policy, Spain.

Ferrer, Antonia. Información en la empresa para innovar y competir. En: El profesional de la información, 2008, v. 17, n. 5, septiembre-octubre, pp. 481-486.

DOI: 10.3145/epi.2008.sep.01

\section{Introducción}

En las dos últimas décadas se ha extendido en nuestra sociedad la llamada economía del conocimiento que se caracteriza por la rápida evolución tecnológica y por la globalización de las actividades. En ese entorno cambiante todas las empresas se ven forzadas a ser más competitivas, debiendo aportar productos de mayor valor añadido (o sea, más sofisticados, avanzados, completos, de mejor calidad). Y es ahí donde se sitúa la innovación no sólo como la palabra de moda sino como una dramática necesidad para poder sobrevivir.

Para hacer frente a la nueva dinámica, el Consejo Europeo reunido en Lisboa en marzo de 2000 aprobó un plan de desarrollo llamado Estrategia de Lisboa (también conocida como Agenda de Lisboa o Proceso de Lisboa), en la que se planteó que el conocimiento y la innovación debían ser factores clave para que Europa pudiera mantener su ventaja competitiva.

Tanto la Estrategia de Lisboa como los resultados del posterior Consejo Europeo celebrado en Barcelona en 2002 marcaron como reto alcanzar en 2010 un gasto de I+D igual al 3\% del PIB, especificando que dos tercios de este gasto debían ser financiados por las empresas. Nos estamos acercando a la fecha y este objetivo, que han venido recogido en distintos documentos relacionados con la política europea de competitividad, aún no se ha cumplido: según el último informe Cotec nuestro país sólo gastó el 1,2\% del PIB en I+D. Por ello resulta obligado que la innovación esté cada vez más presente en las políticas de los gobiernos nacional y autonómicos de nuestro país.

En la proporción de la inversión de las empresas también vamos mal: un 53,8\% del total (la media de la 
Unión Europea es $62,6 \%$, con la meta mencionada de llegar al 66\%).

Según el Índice Sintético de Innovación (SII) elaborado por la Comisión Europea para comparar la innovación entre los estados miembro, España se encuentra en el grupo de los moderadamente innovadores ${ }^{1}$ junto a Estonia, Austria, Noruega, República Checa, Eslovenia, Italia y Chipre. Según este índice en 2007 EUA y Japón se encuentran muy por delante de la UE en materia de innovación² (pág. 72).

Todos estos datos evidencian que es crucial potenciar la innovación.

\section{Concepto de innovación}

Pero, ¿qué se entiende por innovación? En el número de marzo-abril de El profesional de la información Roser Lozano nos hace un planteamiento muy claro de lo que supone la innovación para las bibliotecas públicas. Aquí haremos una revisión del concepto para la empresa y la importancia que tiene la información para poder innovar.

La evolución del concepto de innovación a lo largo de los últimos años puede seguirse a través de las distintas ediciones del Manual de Oslo ${ }^{3}$. En la primera únicamente se consideraba como innovación la industrial, en la segunda se amplía el marco teórico de innovación a un mayor número de empresas centrándose en productos y procesos, y en la tercera se incorpora el sector servicios. La consideración de factores no tecnológicos en los procesos de innovación no es algo nuevo, pues aparecen ya en el Libro verde de la innovación elaborado por la Unión Europea en 1995.

El capital relacional ${ }^{4}$ (la capacidad de establecer vínculos) es un elemento clave para la innovación en una empresa, pues de los contactos depende su acceso a las fuentes de información y conocimiento, tecnologías, buenas prácticas, recursos económicos y humanos.

Según se presenta en la tabla adjunta, se identifican tres tipos de fuentes o relaciones para la transferencia del conocimiento y la tecnología:

- Fuentes de información.

- Adquisición de tecnología y conocimiento. Patentes, licencias, servicios de consultoría y contratación de personal que aporte nuevo conocimiento.

- Cooperación para la innovación. Participación activa con otras empresas o instituciones públicas de investigación en actividades de innovación ${ }^{3}$ (pág. 28).

La captación de señales o información del exterior permite introducir procesos de innovación en la organización.
Por ello nos planteamos una de las cuestiones habituales en nuestro entorno: ¿realmente consumen información las empresas españolas? Existen pocos trabajos recientes que midan el uso de la información en la empresa española. Únicamente conocemos a través de los estudios sectoriales de la Asociación de Empresas de Tecnologías de la Información y Comunicaciones de España (Aetic), Telefónica o los datos recogidos por el Instituto Nacional de Estadística (INE) que las empresas utilizan la Red principalmente para la búsqueda de información (usando prioritariamente Google) por encima de las transacciones bancarias y operaciones con la administración.

Está claro pues, que las empresas tienen necesidad de información, pero que la información que consumen sea de calidad es lo que no sabemos. Las búsquedas se suelen hacer de un modo no estructurado, poco sistemático y por personal no especializado. De hecho, cualquiera se siente capacitado para la búsqueda de información dado que el procedimiento aparece como muy sencillo, pero muy pocas personas saben valorar los resultados.

\section{Uso de información para la innovación}

Ante la actual sobreabundancia de información, que llega a provocar frustración y desasosiego, las empresas innovadoras destacan por saber captar selectivamente la importante para ellas, la que necesitan para prosperar.

En la tabla anterior ya se han indicado varios tipos de fuentes generales de información. Siguiendo a Muñoz-Cañavate ${ }^{5}$, las empresas bien informadas consultan estos tipos específicos:

- Directorios de empresas y ejecutivos.

- Catálogos de productos.

- Directorios de administraciones públicas y cargos políticos.

- Informes comerciales.

- Medios de comunicación.

- Indicadores del entorno (estadísticas, informes económicos y datos político-sociales).

- Información de los mercados financieros (créditos, renta fija, renta variable, materias primas, derivados).

- Ayudas, concursos públicos y oportunidades comerciales.

- Estudios de mercado y estudios sectoriales.

- Información sobre I+D+I (tecnológica -patentes, modelos industriales y marcas-, literatura no convencional, otras fuentes primarias -monografías y publicaciones periódicas-). 


\begin{tabular}{|c|c|c|c|}
\hline & $\begin{array}{l}\text { Fuentes de } \\
\text { información }\end{array}$ & $\begin{array}{l}\text { Fuentes para la } \\
\text { adquisición de } \\
\text { conocimiento y } \\
\text { tecnología }\end{array}$ & $\begin{array}{c}\text { Participantes } \\
\text { en una } \\
\text { acción de } \\
\text { cooperación }\end{array}$ \\
\hline \multicolumn{4}{|l|}{ Fuentes internas a la empresa } \\
\hline $\mathrm{I}+\mathrm{D}$ & $*$ & & \\
\hline Producción & $*$ & & \\
\hline Marketing & $*$ & & \\
\hline Distribución & $*$ & & \\
\hline Otras empresas del grupo & $*$ & $*$ & $*$ \\
\hline \multicolumn{4}{|l|}{ Fuentes externas en el mercado } \\
\hline Competidores & $*$ & $*$ & $*$ \\
\hline Otras empresas del sector & $*$ & $*$ & $*$ \\
\hline Clientes o usuarios & $*$ & & $*$ \\
\hline Expertos/consultoras & & $*$ & $*$ \\
\hline $\begin{array}{l}\text { Proveedores de equipos, materiales, componentes, } \\
\text { software o servicios }\end{array}$ & $*$ & $*$ & * \\
\hline Laboratorios comerciales & $*$ & $*$ & $*$ \\
\hline \multicolumn{4}{|l|}{ Fuentes del sector público } \\
\hline $\begin{array}{l}\text { Universidades y otros establecimientos de educación } \\
\text { superior }\end{array}$ & $*$ & * & $*$ \\
\hline Institutos de investigación gubernamentales/públicos & $*$ & $*$ & $*$ \\
\hline Institutos de investigación privados sin ánimo de lucro & $*$ & * & $*$ \\
\hline $\begin{array}{l}\text { Servicios especializados de apoyo a la innovación } \\
\text { públicos/paraestatales }\end{array}$ & $*$ & $*$ & $*$ \\
\hline \multicolumn{4}{|l|}{ Fuentes de información general } \\
\hline Difusión de patentes & & $*$ & \\
\hline $\begin{array}{l}\text { Conferencias, reuniones, documentación sobre el sector } \\
\text { y estudios profesionales }\end{array}$ & $*$ & & \\
\hline Ferias y exposiciones & & $*$ & \\
\hline Asociaciones profesionales, sindicatos & & $*$ & \\
\hline Otras asociaciones locales & $*$ & & \\
\hline Contactos o redes informales & $*$ & & \\
\hline Estándares y agencias de normalización & $*$ & & \\
\hline Normas públicas (medio ambiente, seguridad, etc.) & $*$ & & \\
\hline
\end{tabular}

Fuente: Manual de Oslo ${ }^{3}$ pág. 94

Tabla. Fuentes para la transformación del conocimiento y de la tecnología

- Legislación, jurispridencia y normativas.

La actividad de informarse de cara a saber actuar y poder tomar decisiones tácticas y estratégicas acertadas se denomina vigilancia o inteligencia. Palop y Vicente $^{6}$ distinguen entre cuatro tipos:

- tecnológica, o centrada en el seguimiento de los avances del estado de la técnica y en particular de la tecnológica y de las oportunidades que genera;

- competitiva, que implica un análisis y seguimiento de los competidores actuales, potenciales y de aquellos con productos sustitutivos;

- comercial, sobre clientes y proveedores; y

- entorno, sobre aspectos sociales, legales, medioambientales y culturales.

Otros autores españoles han publicado también buenos trabajos sobre inteligencia empresarial ${ }^{7,8,9}$.

En un monográfico de la Fecyt sobre innovación ${ }^{10}$,
Robert J. Mockler señala que una de las mayores carencias en las empresas proviene del análisis de la información, su almacenamiento y distribución. Es decir, el problema no es hallar información sino todo lo que se relaciona con maximizar el aprovechamiento de la obtenida.

La búsqueda en bases de datos de prestigio científico-técnicas y de patentes (generalmente de pago) tiene la gran ventaja de asegurar un cierto grado mínimo de calidad en cuanto los documentos que han sido recogidos en ellas (aunque la calidad de los documentos puede no servir para nada si luego no se sabe hacer una buena búsqueda para seleccionar exactamente los que se precisan $)^{11}$. El estudio de las patentes solicitadas por las empresas para registrar sus invenciones permite conocer la evolución de la innovación en las mismas, y es la base de la vigilancia tecnológica competitiva.

Si bien está bastante claro que los investigadores en general suelen usar las bases de datos científico-téc- 
nicas para conocer los avances en su campo de conocimiento, nos planteamos la cuestión de si esos investigadores trabajan en lo que representa la mayoría de la empresa española: la pyme ${ }^{12}$.

Según una nota de prensa del $I N E^{13}$, de julio de 2008 (con datos referidos a $2006^{14}$ ), la mayor parte de los doctores-investigadores (un 44,4\%) trabaja en la enseñanza superior, otro $35,8 \%$ en la administración pública, el $15,7 \%$ en empresas y el resto de $4,1 \%$ en instituciones privadas sin ánimo de lucro. Si la innovación se encuentra vinculada a la investigación, estos datos nos dan una pista de por qué únicamente el $15,6 \%$ de la pyme inno$\mathrm{va}^{15} ; \mathrm{y}$, siguiendo nuestro razonamiento, consume menos información de prestigio. Son las grandes empresas las que tienen acceso a la información de los distribuidores de bases de datos y publicaciones científicas reconocidas, y crean sistemas de información propios para sus líneas estratégicas de negocios.

Algunos de los motivos por los que las empresas no hacen uso de las bases de datos especializadas son conocidos y tienen su raíz en la escasa cultura informacional que tiene el empresariado. Una de las causas es no conceder el debido valor a la información y, en consecuencia, encontrarla demasiado "cara"; muchos de los eventuales consumidores de información consideran que ésta debe ser de acceso gratuito. Por otro lado también hay que decir que las bases de datos que se comercializan no son las ideales para las pymes españolas:

- no se adaptan específicamente a las necesidades de la empresa;

- están en inglés, otro de los grandes handicaps españoles;

En este contexto y a largo plazo, el movimiento Open Access puede ser una alternativa para fomentar el uso de información de calidad en las empresas, y en especial en las pymes. Si la publicación de la información científica es también costeada por las administraciones (al mismo tiempo que subvenciona la investigación), ello permitirá que las empresas de menor tamaño puedan acceder a los avances científicos en igualdad de condiciones que las que tienen mayor poder adquisitivo.

\section{Medidas de apoyo a la innovación}

A lo largo de 2007 se publicaron medidas para fomentar la innovación en las empresas, pero conocer el gasto público dedicado específicamente a ello es difícil ya que en las estadísticas del INE los datos de innovación aparecen conjuntamente con los de investigación y desarrollo. Todas estas facetas suelen ir unidas en el discurrir natural del proceso de innovación.

Existe un hecho claro y es que en los últimos años los recursos económicos públicos para financiar la innovación en España se han incrementando. A pesar de ello, según el último informe de la fundación Cotec de junio 2008, los resultados obtenidos aún no son satisfactorios, ni tampoco el entorno en el que se produce la innovación. En algunos países de la $O C D E$ que tienen una amplia trayectoria en innovación, ésta es financiada por el sector empresarial, estando por ello claramente orientada a la producción. En España la investigación aplicada (vinculada al registro de patentes, transferencia de tecnología, etc.) se encuentra muy por detrás del resto de países europeos. Uno de los motivos es que las relaciones universidad-empresa están poco desarrolladas, debido al desajuste existente entre la formación que se ofrece y lo que demandan las empresas para innovar $^{16}$.

Únicamente las grandes empresas y las multinacionales tienen departamentos dedicados a la innovación, cuyos fondos a menudo proceden de la sede central. Parte de la financiación suele provenir de las distintas iniciativas de fomento de la innovación de carácter europeo, nacional y autonómico.

\section{Fuentes de financiación}

\section{- Ejecución directa de la I+D por parte de las entidades públicas}

Se mide con la información que proviene de los organismos que ejecutan la I+D: centros públicos y universidades. Durante 2006 el gasto en I+D del sector empresarial ha superado al del sector público, siendo un $55,7 \%$ del total ${ }^{3}$ (pág. 156).

\section{- Presupuestos generales del Estado}

La importancia de la innovación como motor de la economía se ha recogido en los presupuestos generales del Estado y en los recursos destinados a la I+D+I. Muestra de ello es el programa Ingenio 2010, a través del cual se organizan distintas actuaciones relacionadas con la I+D+I. Los presupuestos generales del Estado han destinado para el año 2008 la cantidad de 9.437,9 MEUR, lo que representa un incremento del $26,3 \%$ con relación al del año anterior ${ }^{3}$ (pág. 162). 3.046 MEUR se dedican a financiar con créditos y subvenciones las actividades de $\mathrm{I}+\mathrm{D}+\mathrm{I}$ de las empresas privadas (un incremento del 19,3\%).

\section{- Plan Nacional de I+D (2004-2007)}

Se concibió como un mecanismo integrador de las diferentes actuaciones públicas gestionadas por los departamentos ministeriales con competencia en ciencia y tecnología. En este plan se tomó en consideración el Nuevo Espacio de Investigación y el cada vez mayor protagonismo de los planes autonómicos de I+D+I. De todos sus programas, según datos de la Fecyt de febrero de 2008 , sólo un 4\% se dedicó al apoyo de la competitividad empresarial. 


\section{- Plan Nacional de I+D (2008-2011)}

Como es lógico, todavía no se tienen resultados. Pasa del plan basado en áreas temáticas a uno basado en instrumentos que son la respuesta de la Administración Pública a los objetivos estratégicos y operativos fijados en la Estrategia Nacional de Ciencia y Tecnología (Encyt). Pretende "situar a España a la vanguardia del conocimiento; promover un tejido empresarial altamente competitivo; desarrollar una política integral de ciencia, tecnología e innovación; imbricar los ámbitos regionales en el sistema de ciencia y tecnología; avanzar en la dimensión internacional como base del salto cualitativo del sistema; conseguir un entorno favorable a la inversión en I+D+I; fomentar la cultura científica y tecnológica de la sociedad". Todos estos objetivos se desarrollan en cuatro áreas temáticas y 6 líneas instrumentales ${ }^{17}$.

\section{- Programa Ingenio 2010}

Nacido en 2005 con el objetivo de alcanzar el 2\% del PIB destinado a I+D en 2010. Tiene tres subprogramas principales:

\section{- Cenit}

Busca incrementar la capacidad científico-técnica de las empresas y grupos de investigación nacionales, y extender la cultura de participación y cooperación en investigación y desarrollo tecnológico, y preparar a los consorcios para que participen en proyectos internacionales. En este programa se han impulsado los proyectos de I+D de mayor envergadura. Los consorcios de estos proyectos deben estar formados por al menos cuatro empresas, dos de ellas medianas o grandes y dos pymes, junto a dos organismos de investigación.

\section{- Consolider}

Conseguir la excelencia investigadora a través del incremento en la cooperación entre investigadores, formando para ello grandes grupos de investigación.

\section{- Plan Avanza}

Para acelerar el desarrollo de la sociedad del conocimiento mediante la capacitación tecnológica de la ciudadanía. Promueve la creación de redes sociales, impulsa la industria de creación de contenidos digitales, incrementa el número de pymes digitales, eleva la calidad de la I+D empresarial, moderniza los servicios públicos y fomenta el número de infraestructuras de banda ancha. Su presupuesto se divide en cuatro áreas:

- ciudadanía digital,

- economía digital (con el 63\% del presupuesto total dedicado al fomento de la competitividad de las empresas),

- nuevo contexto digital,

- servicios públicos digitales.
Es en este Plan donde el sector de la información puede jugar un papel destacado en sus distintas áreas de actuación, todas ellas muy vinculadas con el sector de la información, organización del conocimiento y nuevos servicios en la sociedad del conocimiento.

\section{- Programa EuroIngenio}

Creado para activar la participación de las empresas españolas en el VII Programa Marco. Éste tiene cinco apartados: EuroCiencia, InnoEuropa, EuroSalud, TecnoEuropa, Fondo EuroIngenio. Este último es un fondo territorial que comenzó a funcionar en 2007 y que aporta recursos para la inversión en I+D+I e infraestructuras para que las comunidades incrementen su participación en el Espacio Europeo de Investigación.

\section{- Incentivos europeos para la innovación}

Aquí se encuentran el VII Programa Marco de I+D, el Programa Marco para la Innovación y la Competitividad, el European Research Council, y el Fondo Tecnológico.

Según datos del CDTI, en febrero de 2008 el VII Programa Marco ha supuesto un retorno a España de 237,9 MEUR. Han participado más de 1.300 entidades, de las cuales 774 son empresas grandes y 658 pymes. Los mayores retornos han sido en el sector de las tecnologías de la información y las comunicaciones, nanotecnologías, materiales y producción.

\section{- Otros programas internacionales}

Eureka, Cyted, Iberoeka, Eiroforum.

Por desgracia, todas estas medidas de fomento de la investigación, desarrollo e innovación tienen una serie de trabas burocráticas que ralentizan considerablemente la concesión de ayudas. Según la evaluación de la Fecyt del Plan Nacional $I+D+I$ y la iniciativa Ingenio 2010 de 2006, el tiempo medio transcurrido entre la publicación de la convocatoria hasta su resolución ha sido de 10 meses. Esto muestra la dificultad existente para la buena ejecución de los proyectos concedidos, donde en bastantes casos no se puede cumplir la programación efectuada ya que prácticamente coincide la fase de adjudicación del proyecto con la de justificación del mismo. El retraso implica que los investigadores deban arriesgarse a comenzar la investigación sin la dotación de recursos suficientes en unos casos, en otros que renuncien a parte de la subvención para poder acometer en el periodo pactado algunos de los objetivos, o que se realice la investigación sin la planificación adecuada para poder beneficiarse del total de la financiación.

Según el informe Cotec es necesario encontrar soluciones para que estas empresas no desaprovechen las ideas innovadoras por no poder llevarlas a término debido a dificultades de financiación en la etapa de in- 
vestigación y desarrollo. En este contexto las empresas deben recurrir a los instrumentos de financiación de la política comunitaria, a los fondos de cohesión y a las ayudas del Plan Avanza.

Existen otras medidas, como la Neotec, con el objetivo de apoyar la creación y consolidación de empresas de base tecnológica en España. Cuenta con instrumentos que facilitan la vida a emprendedores tecnológicos desde que conciben su idea empresarial hasta que pueden convertirla en una empresa viable.

\section{Recursos humanos dedicados a la innovación y evolución de la producción científica}

El capital humano dedicado a innovación es uno de los indicadores más representativos para medirla. Si continuamos con los datos aportados por el informe Cotec se observa cómo se ha incrementado, llegando en 2006 a representar el 5,9\% de la población ocupada.

Otro indicador representativo y a cuyo análisis se dedica una parte importante de los científicos de nuestra profesión biblio-documental, es la evolución de la producción científica española. Ésta se ha incrementado notablemente, prácticamente duplicándose desde 1995 a 2006, pasando de 18.253 artículos a 36.840. Este número representa únicamente un $3,1 \%$ de la producción mundial, lo que continúa siendo poco significativo. Aunque el dato es favorable, el esfuerzo es considerado aún insuficiente. La mayor parte de la producción científica parte de los centros de investigación y de las universidades, que es donde trabaja la mayoría de la población investigadora.

Ello muestra uno de los problemas persistentes en nuestro país: la desvinculación entre los sistemas de formación y las necesidades de la empresa, y la falta de consideración por parte de las empresas de la innovación como factor clave de su competitividad.

Otro elemento de análisis y estudio son las bases de datos de patentes. El número de patentes registradas por las empresas españolas es considerablemente inferior al del resto de los países de la $O C D E$.

Todos estos datos nos muestran cómo la tarea de innovar es aún una asignatura pendiente por parte de las empresas españolas, las cuales, como primera medida deberían aumentar su cultura informacional y concienciarse de que los servicios de información son vitales para ellas.

\section{Notas y bibliografía}

1. El SII diferencia cuatro grupos: los líderes de innovación (Suecia, Suiza, Finlandia, Israel, Dinamarca, Japón, Reino Unido y Estados Unidos), los seguidores (Luxemburgo, Islandia, Irlanda, Austria, Holanda, Francia, Bélgica y Canadá), los moderadamente innovadores (España, Estonia, Aus- tralia, Noruega, República Checa, Eslovenia, Italia y Chipre), y en progreso (Malta, Lituania, Hungría)

2. Fundación Cotec para la Innovación Tecnológica. Tecnología e innovación en España. Informe Cotec 2008. Madrid: Fundación Cotec para la Innovación Tecnológica, 2008, 978-84-95336-82-8.

3. Manual de Oslo: Guía para la recogida e interpretación de datos sobre innovación. Madrid: Grupo Tragsa, 2005. ISBN 978-84-95336-82-8.

Este documento de directrices, que pertenece a la "familia Frascati" de metodologías para la creación de indicadores de la OCDE, es el más utilizado para conocer las actividades de innovación en la empresa.

4. Sánchez M., Paloma; Castrillo, Rocío. "La tercera edición del Manual de Oslo: cambios e implicaciones. Una perspectiva de capital intelectual. En: Revista $I+D, 2006$, marzo-abril, n. 35, pp. 1-16. Consultado en: 2006-2008.

http://www.madrimasd.org/revista/revista35/aula/aula1.asp

5. Muñoz-Cañavate, Antonio. Sistemas de información en las empresas [on line]. Hipertext.net, n. 1, 2003. ISSN 1695-5498.

http://www.hipertext.net

6. Palop, Fernando; Vicente, José-Miguel. Vigilancia tecnológica e inteligencia competitiva. Su potencial para la empresa española. Madrid: Cotec, 1999.

7. Rey-Vázquez, Lara. "Ferroatlántica I+D y la vigilancia tecnológica". En: El profesional de la información, 2006, noviembre-diciembre, v. 15, n. 6, pp. 420-425.

8. Muñoz-Durán, Javier; Marín-Martínez, María; Vallejo-Triano, José "La vigilancia tecnológica en la gestión de proyectos de $\mathrm{I}+\mathrm{D}+\mathrm{i}$ : recursos y herramientas". En: El profesional de la información, 2006, noviembre-diciembre, v. 15 , n. 6, pp. 411-419.

9. Giménez-Toledo, Elea; Román-Román, Adelaida. "Vigilancia tecnológica e inteligencia competitiva". En: El profesional de la información, 2001, vol. 10, n. 5 , p. 11-20.

10. Mockler, Robert J. Vigilancia tecnológica e inteligencia competitiva. Consultado en: 01-07-2008.

http://www.fecyt.es/especiales/vigilancia_tecnologica/

11. The role of information in innovation. Consultado en: 01-07-2008.

http://www.ei.org/documents/Innovation_Brochure.pdf

12. Ferrer, Antonia; Peset, Fernanda. "Análisis de los directorios privados de información empresarial española". En: El profesional de la información, 2007, mayo-junio, v. 16, n. 3, pp. 243-257.

13. Encuesta sobre recursos humanos en ciencia y tecnología 2006. Resultados provisionales (Nota de prensa), 15 de julio de 2008

http://www.ine.es/prensa/np509.pdf

14. Encuesta de recursos humanos en ciencia y tecnología. Consultada el 11-08-2008.

http://www.ine.es/inebmenu/mnu_imasd.htm

15. Encuesta sobre innovación tecnológica en las empresas 2006. Actividades para la innovación tecnológica 2006: Empresas con actividades innovadoras por ramas de actividad, tipo de indicador y tamaño de empresa. Consultada en 11-08-2008.

http://www.ine.es/inebmenu/mnu_imasd.htm

16. Duart, Josep M.; Lara, Pablo; Salomón, Lourdes (2007). "La Universitat Oberta de Catalunya (UOC): Innovación educativa y tecnológica en educación superior". Revista iberoamericana de educación a distancia, $\mathrm{n}$. 9, pp. 315-345. ISSN: 1138-2783.

17. Para más información consultar la web: http://www.plannacionalidi.es/

Antonia Ferrer, Universidad Politécnica de Valencia, Departamento de Comunicación Audiovisual, Documentación e Historia del Arte (Dcadha), Camino de Vera $s / n, 46022$ Valencia.

anfersa@upv.es 